\title{
Research on the Development Model of Modern Leisure Farm
}

\author{
Qian Luo ${ }^{1, a}$ \\ ${ }^{1}$ Haikou College of Economics, Haikou, Hainan, China, 570203 \\ aemail,
}

Keywords: Modern; Leisure Farm; Special Development Model

\begin{abstract}
At present, the process of the development of socialist market economy in China has accelerated the development of urbanization in a certain extent. The process of urbanization has promoted the transformation of urban and rural construction pattern to a certain extent, which caused the corresponding changes in rural and urban areas, how to combine the development of agriculture for the development of modern leisure farms, is the focus of the relevant local research. This paper explores the design and planning methods of modern leisure specialty agricultural park under the background of landscape ecology development from the basic characteristics of modern leisure farm characteristic development mode at this stage. This paper analyzes the current thinking of the development of the current agricultural park on the basis of the landscape ecological planning, and puts forward relevant planning and design measures on the basis of this, aiming at promoting the comprehensive construction and development of the modern leisure farm in China.
\end{abstract}

\section{Introduction}

With the rapid progress of urbanization in our country at present, the present situation of providing a modern life is also facing the alienation of nature. Most of the people living in the city are eager to return to nature, for the natural life of the situation and more yearning. So the current agricultural production and tourism industry fully integrated is a new landscape design model. The development of modern leisure farm characteristics of the development model to promote the development of agriculture to effectively enhance the productivity, to further shorten the gap between urban and rural areas, which can also be more effective in promoting the comprehensive development of agricultural culture in our country at this stage, which is the current urban and rural development and construction an important part. In the current research and development of modern leisure farm characteristics of the model and the construction of the relevant departments need to explore the relevant issues.

\section{The Analysis of Modern Leisure Farms}

The Basic Concept of Modern Leisure Farm. The modern leisure farms mentioned today refer to the industrialization of modern agriculture, the development of large-scale agriculture on limited resources, the basic agricultural projects or the characteristics of agricultural products as the basic support, to learn more mobile idle on the market capital, through the land transfer and construction management, and finally the original land value expansion, which can effectively promote the local farmers of the actual employment problem, but also the new era of market economy under the background of the development of new models of agricultural development, known as tourism and leisure tourism agriculture

Analysis of the Basic Characteristics of Modern Leisure Farm. Most of the current leisure farms are mainly the development of leisure agriculture as the main content of the current modern leisure agriculture has an important impact on the overall development and the main features are described below. Modern leisure farm will be three industries to effectively combine to establish a new market model, to create a higher economic value, making the leisure agriculture has been fully developed. On the other hand also makes the farmland local characteristics of agricultural products on the supply and marketing cycle of a good way. This will not only expand the local agricultural 
economic value, but also make the quality of agricultural products can be guaranteed.

\section{The Overview of Modern Leisure Farm Ecological Planning Design}

At present, Chinese "modern leisure characteristics of the farm" is a lot of overview and different references have different views, such as "leisure agriculture", "rural characteristics of tourism", "farmhouse" and so on. For the "characteristics of the farm" has not yet formed a systematic summary. This paper summarizes the characteristics of farmland through the current situation of agricultural tourism work and the existing conceptual narrative, and points out that sightseeing agriculture is the main basis for the development of actual agricultural production, and combines education, entertainment and ecology. As one of the development of the park, it but also at this stage of the socialist economic development in the context of agriculture and tourism combined with the new industry [1].

Different places have different natural landscape, the development of modern leisure characteristics of the farm model is also a true combination of the local natural environment and human style, for the agricultural landscape and culture were fully integrated use, so that the maximum local resources, so that people can more close to nature, feel the natural place. This will not only promote the development of rural landscape in Liuzhou District, but also can more effectively promote the development of local agricultural economy, making the agricultural landscape more fully realize their own good value. Many areas of natural geographical location, many of them rich ethnic customs, such as Zhuang's songs, Yao's dance and the Dong's buildings are local characteristics, into the process of agricultural tourism development, to enhance the industry's good competition Force also has an important role. On the basis of maintaining the original land, the corresponding design of the space environment, not only to meet the needs of the development of normal agricultural production, but also the landscape and the tourism industry to fully and effectively combine to achieve the comprehensive development of agricultural landscape [2].

\section{The Existing Problems in the Modern Leisure Farm Characteristics Design}

The Project Area Is Unreasonable. At this stage, most of our country has developed the corresponding agricultural projects according to the natural environment of the field, but the actual success of the project is not a lot. According to the effective analysis of the relevant data, at this stage, many of the local farms in the actual operation of the economic benefits obtained by the lower, most of the projects are in a state of loss. The current design of modern leisure farms and planning, the primary consideration is the site of the problem, the current leisure farm project location mainly in the following areas. The farm set in the city and the outskirts of the edge, close to the surrounding cities, so that can ensure adequate and effective source, the formation of an effective market development relations. Followed by the edge is located in the area, you can through the development of scenic areas as a platform for agricultural tourism to provide adequate source. The last category is the characteristics of the type of tourism, most of these areas are rich in agricultural resources have a good foundation for the development, to carry out more effective and comprehensive services. So the current agricultural landscape planning and design process, to avoid blind construction. From the local existing natural environment resources, make reasonable planning layout of the site, in order to achieve the effectiveness of the design [3].

The Construction Project Lacks Scientific and Effective Evaluation. The current development of leisure and tourism farms also need to pay attention to the comprehensive evaluation of agricultural landscape construction projects, through social practice survey and ecological project quality testing and agricultural landscape construction effects, etc., to establish a sound development indicators, the relevant departments to combine a wide range of Resources on the landscape design and construction projects for scientific and effective evaluation. Good evaluation method not only can promote the establishment of the construction system, but also effectively find the construction project itself, many problems, so as to obtain more effective 
resources to solve the existing problems, making the agricultural landscape planning and design has a clear policy Guidance, so as to better promote the construction of agricultural landscape projects. At present, many of Chinese agricultural landscape development projects are subject to tender after the stereotypes of construction, so the lack of scientific and effective evaluation, and ultimately lead to the construction of various types of unreasonable phenomenon, for the original natural ecological environment caused by the corresponding damage. Such unsuccessful construction projects will not only achieve the initial planned economic benefits, but also make the interests of tourists affected by the corresponding damage to the local farmers' economic income growth and the development of agricultural economy caused a corresponding obstacle.

The Agricultural Landscape Construction Type of Popular, It Lacks of Local Characteristics. In many areas of the agricultural landscape planning and design process, the landscape style and performance situation is too monotonous, making the tourists in the course of the journey to produce tired psychology, the project construction design interaction is also more single, mostly all kinds of picking activities, such activities designed to allow visitors to lose interest in the play, so that the actual effect of the construction of agricultural landscape subject to the corresponding restrictions [4].

\section{Research on the Development Model of Modern Leisure Farm}

Parent-Child Interaction Education Model. Leisure farm both science education and production and management functions, from the overall scale of local agricultural development to consider, the scientific research facilities as a tourist attraction, with a modern perspective on its appearance to be modified, so that visitors can feel more deeply natural and scientific and technological knowledge, science and technology and nature are concentrated in the leisure farm, to extend the development of its industrial chain, so that the overall economic value of the farm to effectively improve.

Health Care Culture Model. With the continuous improvement of material living standards, more social masses began to pursue healthy health, health and pension can be based on the actual landscape of the farm, into the pursuit of modern people's health concept to create a beauty and comfort coexist leisure farm can develop a series of characteristics of holiday development model, making the farm to achieve long-term development.

Tourism Experience Natural Model. At present, according to the natural environment of farming arrangements for the corresponding pastoral landscape, to be able to effectively adapt to the spiritual pursuit of modern people, the pursuit of nature. It can be from the external landscape and tourism leisure and other aspects of planning and planning, set up a series of natural leisure activities. In the farm to carry out a series of public welfare activities, and actively create a good atmosphere of the event, so that visitors can feel the natural simplicity in the farm, so as to deepen the impression of tourists to enhance the overall competitiveness of the farm.

Business Meeting Mode. The current characteristics of leisure farms can be based on their own good conditions as the basis for the establishment of various types of ecological environment-related business activities room, based on business activities and then develop more supporting services, making the customer's needs can be met, so as to promote the farm market competitiveness.

\section{Analysis on the Development Mode of Modern Leisure Farm}

The development of modern leisure farm needs to combine the development of various industries to achieve the full integration of various industries, and agricultural commerce, cultural tourism, industrial production and other aspects of organic integration, which makes the modern leisure characteristics of the farm continue to appreciate. With the rapid development of science and technology in China, the construction of various types of leisure and tourism farms should be integrated into agricultural engineering technology, Internet technology and Internet of things technology. Through the big data effect, the actual quality of agricultural products can be improved, 
thus enhancing the overall competitiveness of the market.

Strengthen the Construction of the Preparatory Work. At present, it is necessary to divide the comprehensive resources of the construction area in a reasonable and effective way according to the local natural environment before carrying out the design work of the modern leisure farm. It can be from the local natural environment resources, economic development situation, natural and cultural aspects of a comprehensive analysis, so as to follow-up construction work to lay a good foundation for the development.

Use Scientific and Effective Evaluation of Landscape Construction to Provide Guidance on the Recommendations. The current relevant departments should focus on the actual situation of landscape construction to improve the landscape evaluation system and landscape assessment indicators. Through the relevant evaluation process to draw the corresponding conclusions, so as to better promote the development of tourism agriculture design work effectively. According to the different characteristics of the landscape can take different evaluation methods, such as AHP, expert evaluation and comprehensive evaluation of the factors [5].

The current level of ecological planning and design work in the analytic hierarchy process is to establish a sound evaluation system, the evaluation of the existence of the various elements of the corresponding indicators, according to the completion of the indicators to conduct a comprehensive evaluation. At this stage, the methods of expert evaluation will be used in many construction projects. The evaluation indexes and the elements in the indicators will be evaluated so that the relevant data can be analyzed comprehensively. Finally, the average score is determined according to the expert's assessment. The fractional description is the result of the evaluation. The final agricultural evaluation index system is a comprehensive analysis of the indicators in each region of the agricultural construction project, summarizes the performance aspects of each influencing factor, and establishes the relevant evaluation system model to better promote the development of the construction project

Make a Reasonable Division on the Layout of the Landscape. The layout of the current agricultural landscape in China needs to be based on local characteristics and the existing resources for effective medical treatment, taking into account the principles of ecological sustainable development. According to the current field of natural resources, the division of agriculture is divided into the following aspects: agricultural production development area, science and technology display area, landscape area, recreation area and other aspects of landscape project layout design. Landscape area design layout should take full account of their own geographical environment factors, give full play to the characteristics of different regions, play the inherent power of landscape construction.

Build Various Types of Construction Projects. At present, there are various types of tourism agriculture in China, and more contact with tourists or tourism and leisure projects. To create a unique project cannot blindly plagiarism construction, such a monotony of the external project form makes the agricultural landscape project cannot reflect the local characteristics and cultural customs, and ultimately will lose the beauty of the farm. It can develop the characteristics of agricultural tourism, fishing, etc. to meet the initial psychological ideas of tourists, industrial innovation and social development will be combined to establish more production sites, leisure and cultural consumption as a whole, making the full integration of production and marketing, access Greater economic benefits.

\section{Conclusion}

Based on the above, the study of the development pattern of the modern leisure farm has an important influence on the development of the agricultural economy. The relevant departments of the current planning and design work to conduct a comprehensive study. This paper analyzes the existing problems in the current landscape design and analyzes it in the light of the situation on the ground. On this basis, it analyzes the solution strategy so as to better promote the rapid development of Chinese current agricultural economy. 


\section{References}

[1] Zhang Li, Li Weidong, Yang Lixian, etc. Modern leisure farm characteristics of the development model [J]. China Agricultural Information, 2016 (12): 151-152.

[2] Zhang Qianxi. Show Yichun leisure agriculture characteristics [J]. Jiangxi Agriculture, 2014 (6): 44-45.

[3] Liu Mingxin. Modern leisure farm planning and design [J]. Urban Architecture, 2013 (22): 52-52, 56

[4] Li Huijuan. On the problems and countermeasures in the construction of leisure farm - Taking Hongping Lok farm and Nanning green field as an example [J]. Large Technology, 2017 (3): 1-2.

[5] Lin Guohua, Zeng Yurong, Lin Qing, etc. From the traditional agriculture to the modern leisure and tourism agriculture - to enhance the development of the Hercynian leisure agriculture industry level strategic thinking [J]. Fujian Forum (Humanities and Social Sciences), 2010 (3): 129-132. 